\title{
On Optimization and Extreme Value Theory
}

JÜRG HÜSLER

Dept. Math. Statistics, University of Bern, Sidlerstr. 5, CH-3012 Bern, Switzerland

PEDRO CRUZ

Department of Mathematics, 3810-193 Aveiro, Portugal

ANDREIA HALL

Department of Mathematics, 3810-193 Aveiro, Portugal

CARLOS M. FONSECA

ECEH, University of the Algarve, Campus de Gambelas, 8000 Faro, Portugal huesler@stat.unibe.ch

jpedro@mat.ua.pt

andreia@mat.ua.pt

cmfonsec@ualg.pt

Received April 29, 2002; Accepted February 11, 2003

Abstract. We present a statistical study of the distribution of the objective value of solutions (outcomes) obtained by stochastic optimizers. Our results are based on three optimization procedures: random search and two evolution strategies.

We study the fit of the outcomes to an extreme value distribution, namely the Weibull distribution through parametric estimation. We discuss the interpretation of the parameters of the estimated extreme value distribution in the context of the optimization problem and suggest that they can be used to characterize the performance of the optimizer.

Keywords: optimizer, extreme values, random search, evolution strategies

AMS 2000 Subject Classification: 60G70, 62L12, 65K05, 90C15

\section{Introduction}

Basically, a stochastic optimizer is an algorithm ruled by probabilistic procedures which aims at finding the optimum point of an objective function. Single-objective optimizers produce one scalar outcome per optimization run, which is the best objective value found within the steps of the run. Local minima and convergence speed are fundamental issues, but also the precision of the optimum value is subject of strong attention.

Stochastic optimizers can be seen as estimators of the optimum value of the objective function. By describing the distribution of optimization outcomes, the performance of the optimizer can be assessed, for instance through the usual measures of estimator qualities such as bias or mean square error (cf. Fonseca and Fleming, 1996). In 
particular, if the optimizer is consistent (approaches the optimum value as the number of internal steps increases to infinity), the endpoint of the distribution will be the optimum value and hence it will be of interest to consider endpoint estimation. Several approaches may be taken and non-parametric ones are of much interest since in most cases very little is known about the objective function, and hence about the distribution of the outcomes.

We will be looking at the distribution of outcomes and also at the best of the outcomes found after several executions (runs). We shall always consider minimization problems since the results are readily adapted to maximization problems. We denote an outcome sample of $k$ independent algorithm executions by $\left\{Y_{i}\right\}, i=1, \ldots, k$. Hence each $Y_{i}$ stands for the minima of the same number $n$ of internal steps of the algorithm. The classical limit theorem for extremes states that minima of iid (independent and identically distributed) random variables should have an extreme value limiting distribution as the sample size tends to $\infty$, after an appropriate linear normalization, e.g. Leadbetter et al. (1983) or Falk et al. (1994). This is true only for certain optimizers, as for instance the random search one. In such a situation, $Y_{i}$ is the minimum of $n$ independent and identically distributed values of the algorithm. If $n$ is large, as in the case of the random search algorithm, then we do expect that the $Y_{i}$ are approximately distributed following an extreme value distribution. This will be observed and derived analytically in Section 3. For finite $n$, we expect to be close to the limiting situation and can estimate the parameter values of the extreme value distribution of $Y_{i}$ by several known methods. This will be investigated in Section 2 by some simulations.

For other optimizers this is not true. The number of internal steps $n$ is possibly not large, and in addition, these steps are dependent. However, the distribution of $M_{k}=\min _{i \leq k}\left\{Y_{i}\right\}$ could be approximated still by an extreme value distribution, if $k$ is large, since the $Y_{i}$ are i.i.d. r.v.s. But in addition, if the sample size $k$ is large, then the smallest order statistics of the $Y_{i}$ are approximately following an extreme value distributions or the (negative) excesses below the $m$-th smallest value follow approximately a generalized Pareto distribution if $m$ is small, see Falk et al. (1994). For such cases we do not have yet analytical results for the empirically observed behavior besides of the given argument. But we present simulation results which supports the details of this statement.

Let us mention the extreme value distribution types for minima: for $\mu \in \mathbb{R}, \sigma>0$

$$
\begin{array}{cll}
\Lambda(x)=1-\exp \left(-\exp \frac{x-\mu}{\sigma}\right) & \text { if } \quad x \in \mathbb{R} \quad \text { (Gumbel) } \\
\Phi_{\alpha}(x)=1-\exp -\left(-\frac{x-\mu}{\sigma}\right)^{-\alpha} & \text { if } \quad x \leq \mu, \alpha>0 \quad \text { (Fréchet) } \\
\Psi_{\alpha}(x)=1-\exp -\left(\frac{x-\mu}{\sigma}\right)^{-\alpha} & \text { if } \quad x \geq \mu, \alpha<0 \quad \text { (Weibull) }
\end{array}
$$

Note that the Fréchet distribution has an infinite left endpoint and its domain of attraction (class of distributions for which the minimum has a limiting Fréchet distribution) only includes distributions with infinite endpoint. On the other hand the Weibull distribution has a finite left endpoint and its domain of attraction only includes distributions with finite endpoint. Moreover, in the Weibull family, the shape parameter $\alpha$ determines the rate of growth of the density towards the endpoint: if $\alpha<-1$ the density 


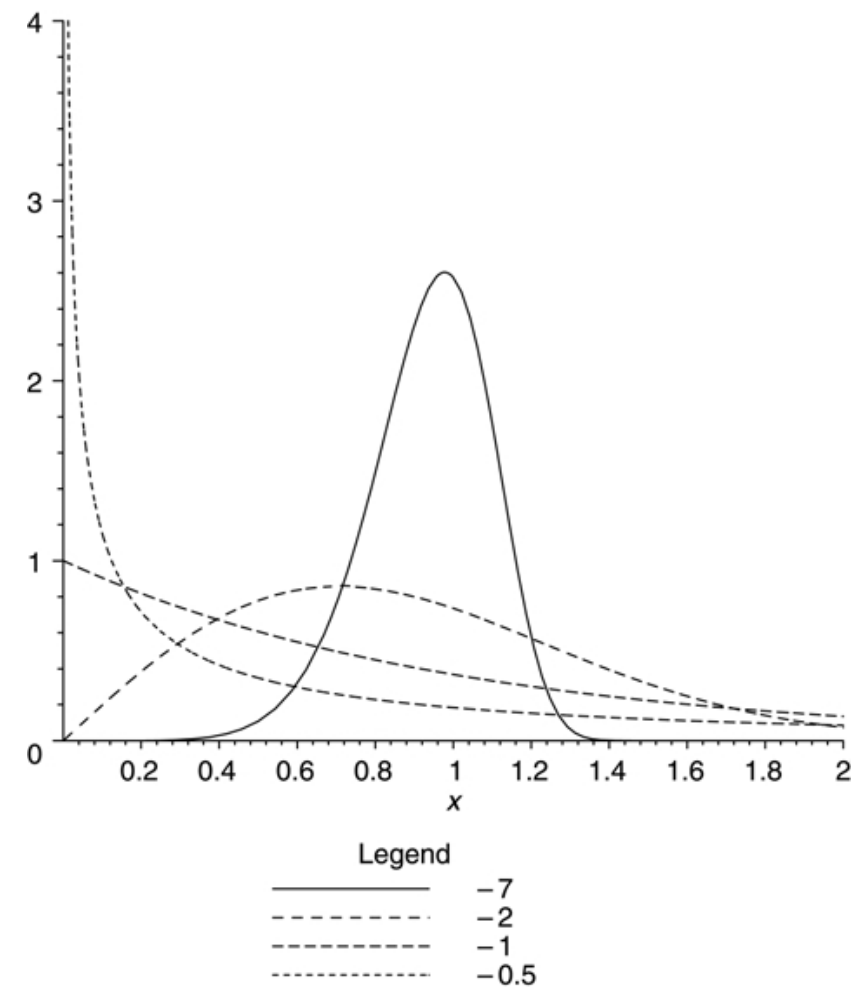

Figure 1. Weibull densities for several $\alpha$ s.

decreases towards zero as it approaches the endpoint, whereas if $-1<\alpha<0$ the density increases monotonically towards infinity. Figure 1 illustrates this behavior. Finally the Gumbel distribution is in between the Fréchet and Weibull distributions having a rather heterogeneous domain of attraction including distributions with both finite and infinite endpoint. Its density looks like a Weibull density with a very negative shape parameter, or equivalently as a Fréchet density with a very large shape parameter.

If an optimizer produces good quality solutions, the outcomes should cluster near the optimum. Thus we should expect the limiting distribution of $M_{k}$ or of $Y_{i}$ (if $n$ is large) to be Weibull. Moreover, the greater the clustering effect is, the smaller (in magnitude) the shape parameter is and the better the solutions are.

If solutions are in the transition state (far from the optimum due to insufficient number of steps) we expect to obtain either a Weibull fit with a very negative $\alpha$, or a Fréchet or Gumbel fit, clearly indicating that the optimum is far away (possibly estimated at infinity).

Thus, it is expected that the shape parameter of the limiting distribution of $M_{k}$ depends on the number $n$ of internal steps. Also the shape parameter can contribute to assess the performance of the optimizer.

We perform a set of simulation studies in order to investigate the use of extreme value modeling in optimization problems. We study the relation between the parameters of the 
distribution and the performance of the optimizer. As mentioned some performances are supported by analytical results.

For each simulation situation considered we estimate the parameters of the extreme value distribution fitting the outcomes. Estimation of the extreme value parameters may be done parametrically or semi-parametrically as described for instance in Falk et al. (1994) or Embrechts et al. (1997). In a parametric perspective the whole sample is used for estimation and assumed to have an extreme value distribution. In a semiparametric perspective, it is assumed that the whole sample may not yet follow an extreme value distribution and only the lower order statistics of the sample are used for estimation since the limiting extreme value distribution characteristics are only contained in the left tail of the distribution of the original variables $Y_{i}$. We applied the parametric approach with maximum likelihood (ML) estimators as implemented in Xtremes (software package included in Reiss and Thomas, 1997). For those situations where ML fails (typically when $-2<\alpha<0$ ) we used an alternative method which is suggested by Smith (1986). In this case the endpoint $\mu$ is estimated by the sample minimum and the other two parameters by ML.

In order to assess the goodness of fit of the Weibull distribution we used the $A^{2}$ test statistic described in Lockhart and Stephens (1994) and developed for situations where the three parameters are unknown.

All simulation studies were performed using Maple, Matlab and Xtremes. An interface platform was developed to automatically transfer data between the different software used.

In this work we consider three distinct optimization procedures: random search and two evolutionary algorithms. We apply the optimization procedures to three specific objective functions: Rosenbrock function $\left((1-x)^{2}+100\left(y-x^{2}\right)^{2}\right)$, simple quadratic function $\left(a x^{2}+b y^{2}, a, b \in \mathbb{R}^{+}\right)$and a function with local minima and multiple global minima $\left(\sin \left(x^{2}+y^{2}\right) /\left(x^{2}+y^{2}\right)\right)$ denoted by sinc.

Section 2 is devoted to random search strategy. Random search has been chosen because of its very low execution time requirement, allowing for many different experiments and serving as motivation for further research. Section 3 contains theoretical results which support the empirical study of the previous section. Section 4 contains experiments using the evolution strategy procedures.

\section{Experiments Using Random Search}

By random search we mean that a prefixed (large) number $n$ of i.i.d. random vectors are generated during an execution run and the objective value is calculated for each one. The outcome will then be the minimum value of the set. We considered bivariate Normal distributions with mean $\xi$ and diagonal covariance matrix $\delta^{2} I$ ( $I$ is the identity matrix) as the distribution of the random vectors.

Using different parameters $\xi$ and $\delta$ we may illustrate and interpret different optimizer behaviors. In the next two paragraphs all simulations performed with a large steps size $n=600$ and an outcome sample size $k=500$. The latter was chosen to reduce sufficiently the simulation error in the statistical estimation. 


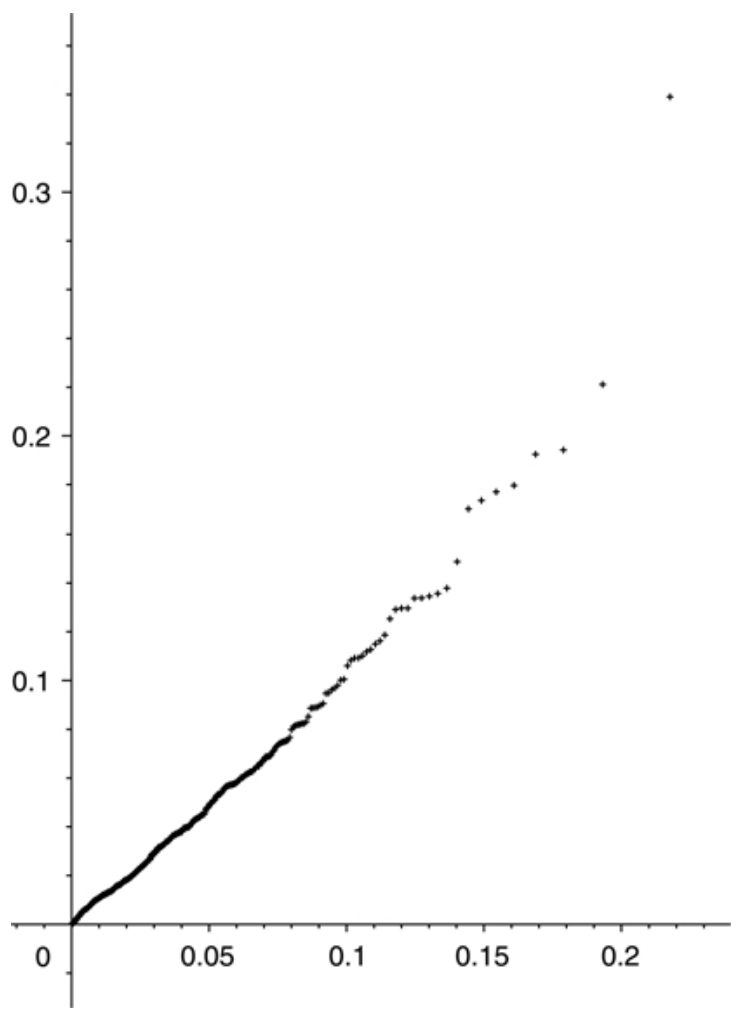

Figure 2. QQ-plot of random search data of case 3 in Table 1, $p$-value $=0.011$ ( $x$-coordinate: Weibull quantiles; $y$-coordinate: sample quantiles).

\subsection{Illustration of the Behavior Near the Optimum}

In order to assure that solutions are near the optimum, random search is performed mostly with $\xi$ equal to the optimum. If $\xi$ is not the optimum, the typical applied situation, then $\delta$ is chosen large enough to guarantee that the optimum is approached. Various $\delta$ values are considered allowing for different behaviors. Table 1 contains a set of representative cases. It reports the estimation of the parameters of an extreme value distribution for each case. The last column refers to the goodness of fit test for the Weibull distribution.

Since each outcome is the best value among $n=600$ i.i.d. values, it is expected that the distribution of the outcomes is itself close to an extreme value distribution. Looking at the $p$-values listed in the table we see that some are rather critical and suggest a bad fit. Figures 2 and 3 contain QQ-plots for cases 3 and 4 (bad and good fit respectively). In Figure 2 we observe one rather large value which influences the $p$-value. This value is not the starting point of the simulation. It is a result of the rather special behavior of the Rosenbrock function. These examples are discussed further in the analytical Section 3, giving the minimum points and minimum value. 
Table 1. Solutions around the optimum.

\begin{tabular}{llllllll}
\hline Case & Function & $\xi$ & $\delta$ & $\hat{\alpha}$ & $\hat{\mu}$ & $\hat{\sigma}$ & $p$-value \\
\hline 1 & Rosenbrock & Optimum & 0.01 & -1.01 & $3.69 \mathrm{e}-11$ & $3.34 \mathrm{e}-6$ & 0.037 \\
2 & Rosenbrock & Optimum & 0.50 & -0.98 & $1.78 \mathrm{e}-07$ & 0.0082 & 0.110 \\
3 & Rosenbrock & Optimum & 1.00 & -0.99 & $1.61 \mathrm{e}-05$ & 0.034 & 0.011 \\
4 & Rosenbrock & $(0,0)$ & 2.00 & -1.00 & $4.52 \mathrm{e}-04$ & 0.172 & 0.500 \\
5 & $0.001\left(x^{2}+y^{2}\right)$ & Optimum & 1.00 & -1.05 & $9.03 \mathrm{e}-09$ & $3.36 \mathrm{e}-6$ & 0.392 \\
6 & $\left(1,000 x^{2}+y^{2}\right)$ & Optimum & 1.00 & -0.96 & $5.04-05$ & 0.103 & 0.457 \\
7 & sinc & $(0,0)$ & 2.00 & -0.49 & -0.2165 & $1.61 \mathrm{e}-05$ & 0.044 \\
\hline
\end{tabular}

\subsection{Illustration of the Behavior Away from the Optimum}

Table 2 contains a set of representative situations where random search is generated away from the optimum.

All cases reported clearly ended in a transition state ( $\hat{\mu}$ is quite far from the optimum) and the estimated $\alpha$ values are very big in magnitude.

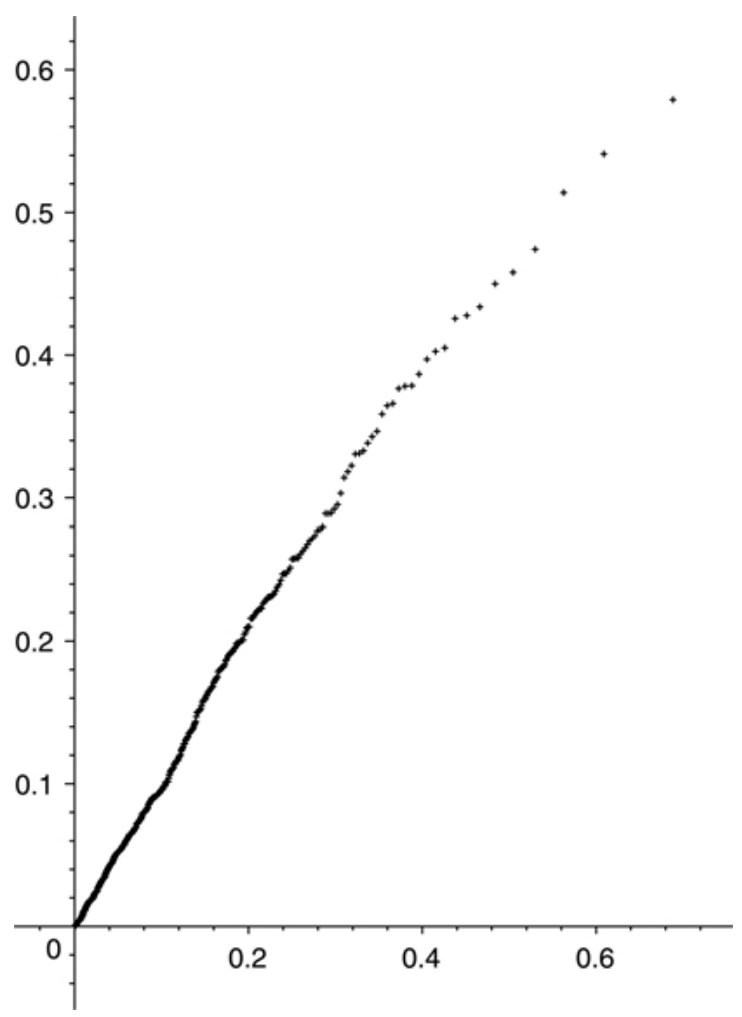

Figure 3. QQ-plot of random search data of case 6 in Table 1, $p$-value $=0.457$ ( $x$-coordinate: Weibull quantiles; $y$-coordinate; sample quantiles). 
Table 2. Transition state solutions.

\begin{tabular}{llllllrr}
\hline Case & Function & $\xi$ & $\delta$ & $\hat{\alpha}$ & $\hat{\mu}$ & $\hat{\sigma}$ & $p$-value \\
\hline 1 & Rosenbrock & $(0,0)$ & 0.01 & -5.94 & 0.908 & 0.0389 & 0.50 \\
2 & $0.001\left(x^{2}+y^{2}\right)$ & $(3,3)$ & 0.10 & -5.76 & 0.014 & 0.0014 & 0.06 \\
3 & $1,000 x^{2}+y^{2}$ & $(3,3)$ & 0.10 & -9.42 & 5712.2 & 1612.1 & 0.05 \\
\hline
\end{tabular}

\section{Analytical Results}

From the experiments described in Section 2.1 it is clear that (excluding the sinc case (7)) the estimated shape parameter $\alpha$ is close to -1 whenever the solutions are near the optimum and very negative when far from the optimum. For the sinc case, $\hat{\alpha}$ is close to -0.5 . Also the $\hat{\alpha}$ values do not seem to be affected by a change of $\delta$ (scale) unless this change modifies the type of solution found.

We shall now state some analytical results which clearly support the empirical results described above for the random search algorithm, letting $n$, the number of steps in an optimization run, grow to infinity. Let $\mathbf{X}_{j}, j=1, \ldots, n$ be i.i.d. random vectors in $\mathbb{R}^{d}$ with $\mathbf{X}_{j} \sim F$ and density $f$. These vectors will denote the points generated within an optimization run which for random search are the steps of the run. Assume that the objective function $g: \mathbb{R}^{d} \rightarrow \mathbb{R}$ has a global minimum $g_{\min }$. If the set of global minimum points is countable we denote the global minimum points by $\mathbf{x}_{l}, 1 \leq l \leq L$ where $L$ is finite or infinite.

Define the domain $A_{u}=\left\{\mathbf{x} \in \mathbb{R}^{d}: 0 \leq g(\mathbf{x})-g_{\text {min }} \leq u\right\}$ for $u$ small. The domain $A_{u}$ can be bounded or unbounded depending on the function $g$. If the domain is bounded, we consider its $d$-dimensional volume $\left|A_{u}\right|$. Furthermore, if $A_{u}$ is concentrated in a lower dimensional subspace $\mathbb{R}^{r}$ of $\mathbb{R}^{d}$, then $\left|A_{u}\right|=0$. It is more reasonable to define the $r$-dimensional volume $\left|A_{u}\right|_{r}$ and consider also the marginal density $f_{r}$ of $f$ in this lower dimensional space related to $A_{u}, r \leq d$. We consider the limiting behavior of the distribution of the outcomes, $Y_{i}$, when $n \rightarrow \infty$.

With the independence of the $\mathbf{X}_{j}$ within each run we get for $Y_{1}=\min _{j \leq n} g\left(\mathbf{X}_{j}\right)$

$$
\begin{aligned}
P\left\{\min _{j \leq n} g\left(\mathbf{X}_{j}\right)>g_{\min }+u\right\} & =P\left\{g\left(\mathbf{X}_{j}\right)>g_{\min }+u, j \leq n\right\} \\
& =\left(1-P\left\{g\left(\mathbf{X}_{1}\right) \leq g_{\min }+u\right\}\right)^{n} \\
& =\left(1-P\left\{\mathbf{X}_{1} \in A_{u}\right\}\right)^{n}=(1-p(u))^{n}
\end{aligned}
$$

We note that the asymptotic behavior of the minimum $\min _{j} g\left(\mathbf{X}_{j}\right)$ depends on the domain $A_{u}$ or more precisely on the probability that $\mathbf{X}$ hits $A_{u}$. This probability $p(u)$ tends usually to 0 as $u \rightarrow 0$, e.g. if the set of global minimum points is countable. The case with $p(u) \rightarrow p>0$ is less interesting for the study of optimizer. If we can normalize this probability $p\left(u_{n}\right)$ with $u_{n}$ such that $n p\left(u_{n}\right) \rightarrow \tau$ for some sequence $u_{n}$, then we may derive limit laws, e.g. for the linearly normalized minimum $\left(\min _{j \leq n} g\left(\mathbf{X}_{j}\right)-g_{\text {min }}\right) / a_{n}$ for some normalization $a_{n}>0$. The following general result is immediate. 
THEOREM 1 Assume that $g$ has a global minimum value $g_{\min }$. Assume that $A_{u}$ and the random vector $\mathbf{X}$ is such that $p(u)=P\left\{\mathbf{X} \in A_{u}\right\} \rightarrow 0$ as $u \rightarrow 0$. If there exists a normalization $u=u_{n}(x)$ such that $n p\left(u_{n}\right) \rightarrow h(x)$, then as $n \rightarrow \infty$

$$
P\left\{\min _{j \leq n} g\left(\mathbf{X}_{j}\right) \leq g_{\min }+u_{n}(x)\right\} \rightarrow 1-\exp (-h(x)) .
$$

If the function $g$ has some isolated global minimum points $\mathbf{x}_{l}, l \leq L$, we can derive a more explicit statement. Assume that the set $A_{u}$ can be split into disjoint sets $A_{u}\left(\mathbf{x}_{l}\right)=\left\{\mathbf{x}: g(\mathbf{x})-g_{\min } \leq u\right.$ and $\left.\left|\mathbf{x}-\mathbf{x}_{l}\right|<\varepsilon\right\}$ for some $\varepsilon>0$ and all sufficiently small $u$. Such cases will be discussed in the examples below.

THEOREM 2 Assume that $g$ has a finite or infinite number of isolated global minimum points $x_{l}, 1 \leq l \leq L \leq \infty$. Assume that each of the disjoint sets $A_{u}\left(\mathbf{x}_{l}\right)$ is bounded and concentrated in $\mathbb{R}^{r}$ with $r \leq d$, for all small $u$ and every $l \leq L$, and that the random vector $\mathbf{X}$ has a positive, uniformly continuous (marginal) density $f_{r}$ at the global minimum points $\mathbf{x}_{l}, l \leq L$, where the marginal density $f_{r}$ corresponds to the space of $A_{u}$. If $u_{n}$ is such that for $l \leq$ L uniformly

$$
n\left|A_{u_{n}}\left(x_{l}\right)\right|_{r} \rightarrow \tau_{l}<\infty \text { with } \sum_{l} f_{r}\left(\mathbf{x}_{l}\right) \tau_{l}<\infty,
$$

then as $n \rightarrow \infty$

$$
P\left\{\min _{j \leq n} g\left(\mathbf{X}_{j}\right) \leq g_{\min }+u_{n}\right\} \rightarrow 1-\exp \left(-\sum_{l \leq L} f_{r}\left(\mathbf{x}_{l}\right) \tau_{l}\right) .
$$

Proof: Since by assumptions the disjoint subdomains $A_{u}\left(x_{l}\right)$ are bounded with a finite $r$-volume $\left|A_{u_{n}}\left(\mathbf{x}_{l}\right)\right|_{r}$, for every $l$, we can approximate

$$
P\left\{X \in A_{u_{n}}\right\} \sim \sum_{1 \leq l \leq L} f_{r}\left(\mathbf{x}_{l}\right)\left|A_{u_{n}}\left(\mathbf{x}_{l}\right)\right|_{r}
$$

by the continuity of the density $f_{r}$. Thus the statement follows by the above derivation (1) and the normalization.

EXAMPLE 1 Assume that $g(x, y)=a x^{2}+b y^{2}$ for some $a, b>0$. Then the minimum of $g$ is at the origin and the bounded domain $A_{u}$ is an ellipsoid in $\mathbb{R}^{2}$. The volume of the ellipsoid $A_{u}$ is $\left|A_{u}\right|=c u$ with constant $c=\pi / \sqrt{a b}$. Hence, select $u_{n}=x /(c n), x>0$. Let $\mathbf{X}$ be any bivariate random vector with continuous density $f$ at the origin. Then by Theorem 2 as $n \rightarrow \infty$

$$
P\left\{\min _{j \leq n} g\left(\mathbf{X}_{j}\right) \leq g_{\min }+\frac{x}{(c n)}\right\} \rightarrow 1-\exp (-f(\mathbf{0}) x)
$$

for $x>0$. Thus $\alpha=-1$ for the Weibull limit distribution. This example can be extended simply to one with a finite number of global minimum points. The function $g(x, y)=a\left(x-c_{1}\right)^{2}\left(x-c_{2}\right)^{2}+b y^{2}$ has two global minimum points $\left(c_{1}, 0\right)$ and $\left(c_{2}, 0\right)$. Similarly, we can replace the term $y^{2}$ by $\left(y-d_{1}\right)^{2}\left(y-d_{2}\right)^{2}$, hence this extended function 
has four global minimum points $\left(c_{k}, d_{l}\right), k, l=1,2$. The result for $\min _{j \leq n} g\left(\mathbf{X}_{j}\right)$ is obvious in these cases.

EXAMPLE 2 We consider now the two-dimensional sinc-function $g(x, y)=\sin \left(x^{2}+y^{2}\right) /$ $\left(x^{2}+y^{2}\right)$. The minimum is attained at points $(x, y)$ with $x^{2}+y^{2}=r_{0}^{2}$ where $r_{0}$ is the smallest positive solution of $r_{0}^{2} \cos r_{0}^{2}=\sin r_{0}^{2}$. Therefore $g_{\min }=\cos r_{0}^{2}$. Hence the domain $A_{u}$ is a ring with center $\mathbf{0}$ and radii $r_{0} \pm \sqrt{2 u / c}$ for some constant $c=\tilde{g}^{\prime \prime}\left(r_{0}\right)+o(1)$ where $\tilde{g}(r)=\left(\sin r^{2}\right) / r^{2}$. Then we need to derive $P\left\{\mathbf{X} \in A_{u}\right\}$ for $u \rightarrow 0$ where $\mathbf{X}$ is bivariate normal with center $\left(\xi_{1}, \xi_{2}\right)$ and covariance matrix $\delta^{2} I$. This is approximately the width of the ring times an integral on the circle with radius $r_{0}$ :

$$
\begin{aligned}
P\left\{\mathbf{X} \in A_{u}\right\} & \sim 2 \sqrt{2 u / c} \int_{0}^{2 \pi} r_{0} \frac{1}{2 \pi \delta^{2}} \exp \left\{-\frac{1}{2 \delta^{2}}\left(r_{0} \cos \phi-\xi_{1}\right)^{2}+\left(r_{0} \sin \phi-\xi_{2}\right)^{2}\right\} d \phi \\
& =D u^{1 / 2}
\end{aligned}
$$

Select now $u_{n}(x)=x /(D n)^{2}$ to get as $n \rightarrow \infty$

$$
P\left\{\min _{j \leq n} g\left(\mathbf{X}_{j}\right) \leq \cos r_{0}^{2}+\frac{x}{(D n)^{2}}\right\} \rightarrow 1-\exp \left(-x^{1 / 2}\right)
$$

for $x>0$. Hence $\alpha=-0.5$ for the Weibull limit distribution.

EXAMPLE 3 Let us now choose $g(x, y)=x^{2}$. This example is by rotation similar to $g(x, y)=(x+y)^{2}$. The set $A_{u}$ in $\mathbb{R}^{2}$ is unbounded: $A_{u}=\{(x, y):|x| \leq \sqrt{u}\}$. But relevant for the minimum is just the first component. If the random vector $\mathbf{X}$ is standard bivariate Gaussian with correlation $\rho$, then $P\left\{\mathbf{X} \in A_{u}\right\}=P\left\{\left|X_{1}\right| \leq \sqrt{u}\right\}=2 \Phi(\sqrt{u})-1$ where $\Phi$ denotes the unit normal distribution. Thus $P\left\{\mathbf{X} \in A_{u}\right\} \sim 2 \sqrt{u / 2 \pi}$ as $u \rightarrow 0$. Hence, select $u_{n}=\pi x /\left(2 n^{2}\right)$ to get by Theorem 1

$$
P\left\{\min _{j \leq n} g\left(\mathbf{X}_{j}\right) \leq \frac{\pi x}{\left(2 n^{2}\right)}\right\} \rightarrow 1-\exp \left(-x^{1 / 2}\right)
$$

for $x>0$ and $\alpha=-0.5$ of the Weibull limit distribution. This shows a case which is not directly included in Theorem 2 . But if we consider $A_{u} \in \mathbb{R}$ being the interval $(-\sqrt{u}, \sqrt{u})$ since $g$ is only a function of $x$, then we can apply the result of Theorem 2 with the onedimensional volume, the length of $A_{u}=2 \sqrt{u}$, and the corresponding marginal density $f_{1}(0)=1 / \sqrt{2 \pi}$.

If the function $g$ is rather regular, i.e. twice continuously differentiable at a unique global minimum point, we can state another general result. If there are finitely many isolated global minimum points, it is obvious how to extend the following result.

THEOREM 3 Assume that $g$ is twice continuously differentiable at the unique global minimum point, say at the origin, and that the random vector $\mathbf{X}$ has a positive continuous (marginal) density $f$ at the origin. If the Hessian matrix $H$ has full rank $d$ and $u_{n}(x)=n^{-2 / d} x$, then as $n \rightarrow \infty$ 


$$
P\left\{\min _{j \leq n} g\left(\mathbf{X}_{j}\right) \leq g_{\min }+n^{-2 / d} x\right\} \rightarrow 1-\exp \left(-x^{d / 2} f(\mathbf{0}) c_{d} \prod_{l \leq d} \lambda_{l}^{-1 / 2}\right),
$$

for any $x>0$, with $c_{d}=\pi^{m} / m !$ for $d=2 m$ even, and $c_{d}=2^{m+1} \pi^{m} /(1 \cdot 3 \cdot 5 \cdot \ldots \cdot d)$ for $d=2 m+1$ odd, the volume of the d-dimensional unit sphere, and $\lambda_{l}$ the positive eigenvalues of $H$.

Proof: By assumptions $g(\mathbf{x})=g_{\min }+\mathbf{x}^{T} H x+o\left(|\mathbf{x}|^{2}\right)$. Hence $A_{u}$ is (approximately) an ellipsoid with a finite $\left|A_{u}\right|$ for all sufficiently small $u$, and we can approximate

$$
P\left\{X \in A_{u_{n}}\right\} \sim f(\mathbf{0})\left|A_{u_{n}}\right| \sim f(\mathbf{0}) c_{d} \prod_{l \leq d} \lambda_{l}^{-1 / 2} u_{n}^{d / 2}
$$

by the continuity of the density $f$ at $\mathbf{0}$ where $c_{d} \prod_{l \leq d} \lambda_{l}^{-1 / 2}$, is the volume of the ellipsoid in $\mathbb{R}^{d}$ with axes $\lambda_{l}^{-1 / 2}$. Thus the statement follows by Theorem 1 and the normalization.

EXAMPLE 4: We consider the generalized Rosenbrock function $g(x, y)=(1-x)^{2}+$ $a\left(y-x^{2}\right)^{2}$ for some $a>0$. In the simulation we used the standard constant $a=100$. The function $g$ has continuous second derivatives, the unique global minimum point is at $(1,1)$. The Hessian matrix is

$$
H=\left(\begin{array}{cc}
8 a+2 & -4 a \\
-4 a & 2 a
\end{array}\right)
$$

with eigenvalues $\lambda_{l}=(10 a-1) \pm \sqrt{100 a^{2}+16 a+1}$ for $l=1$, 2. Thus $\lambda_{1} \lambda_{2}=4 a$. Finally, for any bivariate distribution with continuous density $f$ at $\mathbf{1}$ and normalization $u_{n}(x)=f(\mathbf{1})(8 a / \pi) x n^{-1}$ we have by Theorem 3 for any $x>0$ as $n \rightarrow \infty$

$$
P\left\{\min _{j \leq n} g\left(\mathbf{X}_{j}\right) \leq f(\mathbf{1}) \frac{8 a x}{\pi n}\right\} \rightarrow 1-\exp (-x) .
$$

Hence we find $\alpha=-1$ for the Weibull limit distribution which confirms the findings of Section 2.

\section{Experiments Using Evolution Strategies}

In this section we perform a similar study as in Section 2 but with two evolution strategies.

The first is a simple evolutionary algorithm described by the following scheme:

1. Choose a starting point $(a, b) \leftarrow\left(x_{0}, y_{0}\right)$ and a positive constant $\delta$.

2. Generate 6 bivariate Normal points $\left(a^{i}, b^{i}\right), i=1, \ldots, 6$, with mean $(a, b)$ and covariance matrix $\delta^{2} I$.

3. Let $(a, b) \leftarrow\left(x_{1}, y_{1}\right)$ such that $g\left(x_{1}, y_{1}\right)=\min _{i \leq 6} g\left(a^{i}, b^{i}\right)$.

4. Repeat (2) and (3) a fixed number $n$ of times (steps) and obtain a set of points $\left(x_{j}, y_{j}\right), j=1, \ldots, n$.

5. Return the solution $\left(x_{\text {opt }}, y_{\text {opt }}\right)$ such that $g\left(x_{\text {opt }}, y_{\text {opt }}\right)=\min _{j \leq n} g\left(x_{j}, y_{j}\right)$. 
Table 3. Evolution strategy 1 with $\delta=0.1$.

\begin{tabular}{|c|c|c|c|c|c|c|}
\hline Case & Start Point & Steps & $\hat{\alpha}$ & $\hat{\mu}$ & $\hat{\sigma}$ & $p$-value \\
\hline 1 & $(-1,-1)$ & 100 & -0.652 & $4.23 e-5$ & 0.011 & 0.00 \\
\hline 2 & optimum $(1,1)$ & 50 & -0.783 & $3.57 \mathrm{e}-6$ & 0.0039 & 0.03 \\
\hline
\end{tabular}

Table 4. Evolution strategy 2.

\begin{tabular}{llllllll}
\hline Case & Start Point & Steps & $\delta$ & $\hat{\alpha}$ & $\hat{\mu}$ & $\hat{\sigma}$ & $p$-value \\
\hline 1 & optimum $(1,1)$ & 10 & 0.01 & -0.507 & $1.1 \mathrm{e}-6$ & 0.0608 & 0.19 \\
2 & optimum $(1,1)$ & 10 & 1.00 & -0.200 & $4.5 \mathrm{e}-12$ & 0.0011 & 0.00 \\
3 & $(-1,-1)$ & 10 & 0.01 & -0.530 & $2.1 \mathrm{e}-6$ & 0.0985 & 0.08 \\
4 & $(-1,-1)$ & 50 & 0.01 & -0.477 & $5.0 \mathrm{e}-8$ & 0.0022 & 0.50 \\
\hline
\end{tabular}

The number of "offsprings"' $(a, b)$ in step 2 can also be larger than 6 , but in certain situations 6 is a best choice (see Bäck et al., 1991). Often more offspring points, as 10, are used, resulting in a more stable self-adaption, but also in a larger computing effort.

The second evolution strategy used is denoted by $(1,6)-E S$ in Bäck et al. (1991) and differs from the first by the fact that the variance in step 2 is not constant but rather random and adaptive in order to approach the optimum faster. In this case each of the random points in step 2 has its own covariance matrix $\gamma_{j-1}^{2} e^{Z} I$ where $\gamma_{j-1}^{2}$ is the variance used to generate the previous center $\left(x_{j-1}, y_{j-1}\right)$ and $Z \sim N\left(0, \delta^{2}\right)$.

Obviously, the interal steps and random variables are dependent, thus we do not expect that $Y_{j}$ follows an extreme value distribution for small or moderate $n$.

All the cases reported in Tables 3 and 4 were based on samples of outcomes of size $k=500$ and different number $n$ of steps for the Rosenbrock function.

In most cases the quality of the solutions is quite good as can be seen from the several estimated parameters. Note the smaller values (in magnitude) of $\hat{\alpha}$ when compared to the ones obtained in Section 2.

It is clear that the second evolution strategy performs better than the first. Not only the algorithm is able to reach the optimum in a smaller number $n$ of steps (when starting away from the optimum) but also the shape parameter indicates a greater clustering effect near the optimum.

Figures 4 and 5 show two cases which indicates our conjecture that the smallest of the minima $Y_{j}$ are following well the extreme value behavior of order statistics. The simulated points are almost on a straight line near the minimum.

In general, evolution strategies are expected to perform better than random search. The results we obtained clearly confirm this and also show that the improvement is also reflected in the shape parameter of the distribution of outcomes. Note that some of the estimated $\alpha$ values are closer to zero than in the cases reported previously. As proved in Section 3 random search imposes a natural bound for the shape parameter which cannot be overcome by choosing different scales or starting points. Evolution strategies are able to overcome this bound and produce distributions which cluster much stronger near the optimum, thus producing better solutions. 


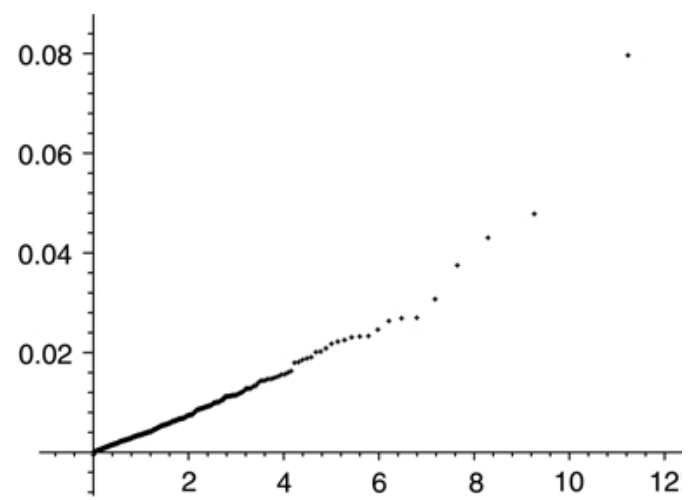

Figure 4. QQ-plot of data of case 2 in Table 3, $p$-value $=0.03$, Weibull distribution for minima with $\alpha=0.783$, ( $x$-coordinate: Weibull quantiles; $y$-coordinate: sample quantiles).

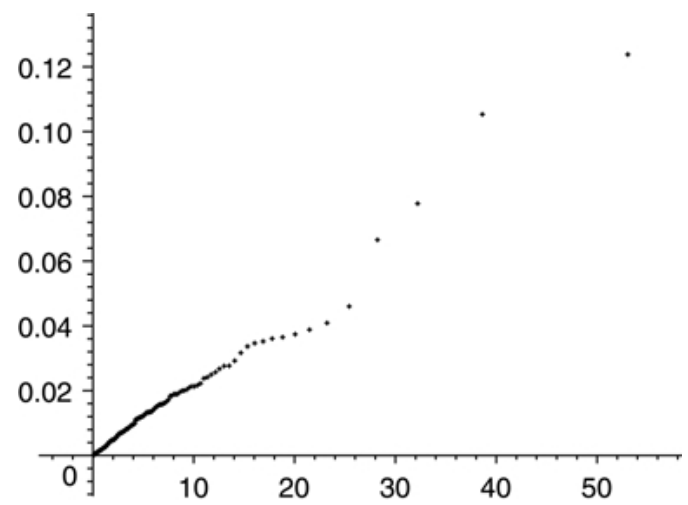

Figure 5. QQ-plot of random search data of case 4 in Table $4, p$-value $=0.50$, Weibull distribution for minima with $\alpha=0.477$, ( $x$-coordinate: Weibull quantiles; $y$-coordinate: sample quantiles).

\section{Acknowledgments}

This research was partially supported by the Swiss National Science Foundation, by Portuguese Foundation for Science and Technology through projects POCTI/MAT/10135/ 98 and POCTI/33477/MAT/2000 and through Plurianual Funding (R\&D Unit "Mathematics and Applications', University of Aveiro).

\section{References}

T. Bäck, F. Hoffmeister, and H.-P. Schwefel. "A Survey of Evolution Strategies. Genetic Algorithms”, in Proceedings of the Fourth International Conference at San Diego, Ca., Morgan Kaufmann: San Mateo, Ca., pp. 2-9, 1991. 
P. Embrechts, C. Klüppelberg, and T. Mikosch, Modelling Extremal Events for Insurance and Finance, SpringerVerlag: Berlin, 1997.

M. Falk, J. Hüsler, and R. D. Reiss, Laws of small numbers: extremes and rare events, DMV-Seminar Series 23, Birkhäuser: Basel and Boston, 1994.

C. M. Fonseca and P. J. Fleming, "On the performance and comparison of stochastic multiobjective optimizers", Lecture Notes in Computer Science, vol. 1141, Springer, pp. 584-593, 1996.

M. R. Leadbetter, G. Lindgren, and H. Rootzén, Extremes and Related Properties of Random Sequences and Processes, Springer-Verlag: New York, 1983.

R. Lockhart and M. Stephens, "Estimation and tests of fit for the three-parameter Weibull distribution," J.R. Statist. Soc. B, vol. 56, pp. 491-500, 1994.

R.-D. Reiss and M. Thomas, Statistical Analysis of Extreme Values with Application to Insurance, Finance, Hydrology and other fields, Birkhäuser: Basel, 1997.

R. L. Smith, "Extreme value theory based on the $r$ largest annual events", J. Hydrology vol. 86, pp. 27-43, 1986. 